\title{
Nonviral Vectors: We Have Come a Long Way
}

\author{
Tyler Goodwin and Leaf Huang ${ }^{1}$ \\ Division of Molecular Pharmaceutics and Center for Nanotechnology in Drug Delivery, Eshelman \\ School of Pharmacy, University of North Carolina at Chapel Hill, Chapel Hill, NC, USA
}

\begin{abstract}
Gene therapy, once thought to be the future of medicine, has reached the beginning stages of exponential growth. Many types of diseases are now being studied and treated in clinical trials through various gene delivery vectors. It appears that the future is here, and gene therapy is just beginning to revolutionize the way patients are treated. However, as promising as these ongoing treatments and clinical trials are, there are many more barriers and challenges that need to be addressed and understood in order to continue this positive growth. Our knowledge of these challenging factors such as gene uptake and expression should be expanded in order to improve existing delivery systems. This chapter will provide a brief overview on recent advances in the field of nonviral vectors for gene therapy as well as point out some novel vectors that have assisted in the extraordinary growth of nonviral gene therapy as we know it today.
\end{abstract}

\section{INTRODUCTION}

The past several decades have shown immense growth in the knowledge of the ability to create and improve nonviral vectors for the delivery of genetic material. This genetic material has great promise as a therapeutic agent against numerous aliments including genetic disorders, chronic and acute diseases, and cancer. Within this field of nonviral vectors, we have produced promising physical methods and chemical vectors for gene delivery consisting of electroporation techniques, cationic lipids, cationic polymers, hybrid lipid polymers, as well as many others. An increased understanding of the field has catalyzed efficiency to new levels in which delivery of plasmid DNA or oligonucleotide into cells can be well characterized and has yielded promising results in preclinical and clinical trials. These vectors have shown to be a promising alternative to viral vectors due to their safety, adaptability, and efficiency in large-scale production. Nonviral vectors have demonstrated their potential to be the next delivery systems of genetic material. They have been shown to exhibit cell specificity through addition of targeting ligands, minimal immune toxicities through addition of inflammatory suppressor molecules, as well as sufficient genetic material release into the cytoplasm of the cell through endosomal destabilization via proton sponge effect or other mechanisms. However, even with these strides, the field of nonviral gene therapy has many areas that need to be addressed, particularly in gene release, nuclear uptake, and expression, which are lagging behind viral vector capabilities. With each

\footnotetext{
${ }^{1}$ Corresponding author: leafh@email.unc.edu.
} 
vector comes advantages and disadvantages, which will be addressed throughout Part I and Part II of this book.

\section{CHEMICAL METHODS}

The chemical methods which deliver genetic material via a vector consisting of cationic lipids (lipoplex), cationic polymers (polyplex), or lipid-polymer hybrids (lipopolyplex) have shown promise. These vectors are being used as a systemic approach to delivering genetic material. Therefore, many challenges need to be addressed in order to improve and generate ideal nonviral vectors. These vectors must overcome barriers which consist of extracellular stability, specific cell targeting, internalization, endosomal escape, nucleotide release, nuclear envelope entry, and genome integration (Figure 1.1) (Hu, Haynes, Wang, Liu, \& Huang, 2013). These first few barriers mentioned seem to have been accomplished to a reasonable level. Multiple vectors have become efficient at achieving long circulation halflife with stable carrier molecules and the addition of hydrophilic moieties such as polyethylene glycol (PEG). The improved cell specificity and internalization with the conjugation of targeting ligands, as well as endosomal escape through the proton sponge effect, have also been achieved with moderate success. By overcoming these initial barriers and being able to deliver genetic material into the cytoplasm of the diseased cell, numerous oligonucleotides, mainly siRNA, are reaching new levels in clinical trials. However, in order to truly reach clinical efficiency in DNA delivery, we must improve intracellular nucleotide release, nuclear entry, and genome integration.

\subsection{Cationic Lipid-Based Nanoparticles (Lipoplex)}

Cationic lipid-based gene delivery (lipofection) was first published by Felgner's group in the late 1980s (Felgner et al., 1987). It has become the most studied and popular of all nonviral gene delivery methods and is discussed further in part I, chapters 2, 3, 4, and 7. The basis for using cationic lipids as a delivery system for negatively charged DNA is that the positively charged hydrophilic head group can condense with the DNA while the hydrophobic tail can form micellar or bilayer structures around the DNA. This complexation of lipids around the DNA has been termed a lipoplex and yields DNA protection against nucleases. There are numerous lipid structures that have been tested in order to find optimal lipids to form a lipoplex structure with DNA. The head groups can vary from primary, secondary, and tertiary amines, or quaternary ammonium salts as well as phosphorus, guanidino, arsenic, imidazole, and pyridinium groups. The hydrophobic tails consist of aliphatic chains which can be unsaturated or saturated and are connected to the hydrophilic head by a linker usually consisting of an ester, ether, carbamate, or amide. Cholesterol, as well as other steroids, is usually included in the formulation of these lipoplexes in order to increase the stability and flexibility of these vectors and have been shown to improve transfection in vivo. All of these components are critical in formulating promising nonviral gene delivery vectors. Varying these components can drastically change the transfection efficiency as well as improve uptake into the cell and release from the endosome. The electrostatic interaction between the negatively charged cellular membrane and the positively charged lipid head groups is vital in achieving higher levels of cellular uptake. The lipid fusion mechanism in which the positively charged vectors fuse with the cellular membrane ultimately resulting in cellular 
uptake of genetic material is promoted by vectors with increased flexibility as well as neutral or helper lipids (colipids) that can assist in this fusion with the cellular membrane (Li \& Szoka, 2007). The fusogenic properties which facilitate cellular uptake are also valuable in the endosomal escape of lipoplexes through membrane destabilization followed by DNA release from the vector into the cytoplasm of the cell. Although the simple early lipoplexes have the capability to deliver genetic material to cells, they have drawbacks which include low transfection, an inability to target specific cells, short half-life, and toxicity due to the positively charged lipids used. Many more details and examples of cationic lipid vectors are discussed in part I, chapters 2, 3, 4, and 7.

To address the short circulation and toxicity issues with cationic lipid vectors, PEG has been introduced to the surface of these vectors in order to shield the positive charge and reduce opsonization from the reticuloendothelial system. The addition of PEG increased circulation time, allowing more time for these vectors to transfect cells (Harvie, Wong, \& Bally, 2000); however, the surface PEG prevents an interaction between the cationic lipoplexes and anionic cell membrane, reducing the overall transfection efficiency. Therefore, in order to increase cellular uptake of these PEGylated lipoplexes, several strategies have been devised. The conjugation of cell-specific targeting ligands to the distal end of PEG, as well as the addition of PEG-lipid conjugates with shorter alkylated chains that can shed off the vector while in circulation over time, have shown promise. The incorporation of chemically sensitive bonds has also improved the shedding of PEG once inside an acidic or reducing environment such as the endosome or cytoplasm (Li \& Szoka, 2007).

Prolonged circulation time and decreased toxicity due to surface modification makes targeted gene delivery to cells located in the interstitial regions possible. Improvements in these nonviral cationic lipid vectors have proved to be promising in gene transfer, especially in the field of siRNA delivery. In addition to its applications in systemic delivery, local DNA and siRNA delivery has shown promise with significant efforts in the delivery of genes directly to the respiratory tract for the treatment of cystic fibrosis, as well as to the cornea and retina for treatment of ocular degenerative diseases (Farjo, Skaggs, Quiambao, Cooper, Naash et al., 2006).

Major preclinical and clinical studies have been completed in the field of cationic lipid gene therapy vectors, but in order for these vectors to truly make a large impact on the medical field, several challenges still lay ahead. Cationic lipids carrying unmethylated CpG DNA have been shown to increase inflammatory responses in the patient (Yew et al., 2000). In addition, quickly dividing cells have shown to have short gene expression due to the DNA dilution over dividing daughter cells. Incorporation of the delivered gene into the cell's genome would allow much more efficient and long-lasting expression of the desired gene. Only when these challenges can be overcome will cationic lipid vectors truly revolutionize the field of gene therapy.

\subsection{Cationic Polymer-Based Nanoparticles (Polyplex)}

Cationic polymer-based nanoparticles, discussed further in part I, chapters 8, 9, and 10, have been an alternative choice to cationic lipids due to their chemical diversity and potential for functionalization through chemical synthesis. Polyplexes have some advantages over 
lipoplexes including low enzymatic degradation, more stability, and greater manipulation of their physical characteristics. Two of the earliest and most used cationic polymers are polyethylenimine (PEI) and poly(L-lysine) (PLL). PLL, which contains cationic lysine residues in physiological $\mathrm{pH}$, is a promising polymer due to its capability to condense DNA, as well as its potential to be conjugated to cell-specific targeting ligands. However, PLL has shown many drawbacks due to a permanent positive charge throughout the life of the polymer in vivo. Some of these drawbacks include low levels of escape from the endosome due to buffering from the cationic amines, as well as high levels of toxicity. In order to address these issues, PLL polymers have incorporated endosomal escape moieties such as chloroquine and have added PEG in order to reduce the toxicity caused from the cationic charges. PLL has shown great promise in the field of ocular gene therapy. The DNA is condensed with the cationic PLL and delivered to the desired site by direct injection of the particles. The compacted DNA nanoparticles seem to have no limit on plasmid DNA size, and at high concentrations have been shown to be safe and effective in human clinical trials, provoking no immune responses (Farjo et al., 2006).

The polymer PEI consists of a secondary amine which is only protonated at a lower $\mathrm{pH}$ which is achieved in the late endosome. This characteristic of PEI is believed to aid in condensation of DNA and endosomal escape through the proposed proton sponge effect. Although these secondary amines seem to play a vital role in gene delivery and expression levels, other studies suggest that the structural properties, degree of branched or linearity, and molecular weight also play a vital role (Wightman et al., 2001). These structural properties may influence the ability of the polymer to deliver the genetic material into the nuclear membrane after endosomal escape. PEI, however, has also been shown to cause high levels of toxicity and therefore, the PEI-PEG block copolymer has been used in order to create a more biocompatible nanoparticle with longer circulation time.

Second-generation polymers are now being introduced into the field of cationic polymers in order to address the drawbacks of PEI and PLL. These new polymers include a poly[(2dimethylamino)ethyl methacrylate](pDMAEMA), poly-arginine containing proteins, poly( $\beta$ amino ester) s, poly lactic-co-glycolic acid (PLGA)-based nanoparticles, carbohydrate-based polymers such as heparin and dextran, and dendrimers (Mintzer \& Simanek, 2009). PLGAbased nanoparticles have been recognized as a potential vector to deliver genes. Research shows that PLGA has an improved safety profile compared to high-molecular weight PEIs and liposomes. Polysaccharides and other carbohydrate-based polymers are also attractive due to high stability, biocompatibility, and biodegradability. These carbohydrate polymers have also been shown to have lower levels of toxicity compared to PEI and PLL. Dendrimers are highly branched spherical structures with a high population of primary, secondary, and tertiary amines. The most common and promising dendrimer with higher levels of transfection is polyamidoamine. It has been shown that the amine groups and the molecular weight greatly impact expression levels. The mechanism in which dendrimers facilitate gene delivery is one such that the primary amine groups enhance DNA cellular uptake by binding DNA, while the more sterically hindered tertiary amine groups promote endosomal escape via the proton sponge effect (Pack, Hoffman, Pun, \& Stayton, 2005). 
Similarly to cationic lipids, the levels of gene expression from these polymers fall short of the levels expressed after viral gene delivery. However, these cationic lipids and polymers show promise in preclinical and clinical trials and in improving our knowledge and understanding of how to deliver genetic material to the nucleus of the cell. As our understanding of the mechanisms between nanoparticles and the cellular/nuclear uptake of these materials increases, as will the efficiency of the nanoparticles we formulate.

\subsection{Hybrid Lipid-Polymer-Based Nanoparticles (Lipopolyplex)}

Hybrid nanoparticles usually consist of a polycation-DNA core with an outer layer shell consisting of lipids. The two main groups are lipid-polycation-DNA (LPD) nanoparticles and multilayered nanoparticles, in which the multilayered nanoparticles are formulated through a layering technique in which cationic polymers and DNA are added sequentially. In most vectors a cationic polymer with the ability to condense DNA is crucial. The main challenge in selecting a cationic polymer is the balance of strong yet reversible electrostatic binding which sufficiently condenses with the anionic DNA backbone, but will release the DNA once cellular/nuclear uptake has occurred. The lipids associated with LPDs can be of two categories: LPDI is referred to when cationic lipids are used, while LPDII is used when anionic lipids are incorporated. The use of cationic lipids can have higher degrees of toxicity, but also improve cellular uptake and endosomal release through the hexagonal fusion with the endosomal membrane. The incorporation of PEG with targeting ligands can also be used to decrease toxicity and improve cell-specific targeting.

These hybrid nanoparticles, such as LCP (mc-CR8C) Gal shown in Table 1.1, express high therapeutic levels of luciferase in the liver of mice (Hu et al., 2013). Although hydrodynamic injections result in an expression level 100 times higher than the LCP vector, it is not necessary to achieve these high levels to have therapeutic effects. Many hybrid nanoparticles are discussed in further detail in part I, chapters 5, 6, and 7. The main challenge still to be addressed, is how to maintain these levels of expression for prolonged periods of time. This may be possible through new findings in which the delivery of genome-editing systems such as zinc-finger nuclease, a transcription activator-like effector nuclease, or clustered regularly interspaced short palindromic repeat-associated system and repair template could allow the integration of the desired genetic material into the cellular genome (Gaj, Gersbach, \& Barbas, 2013).

\section{PHYSICAL METHODS}

Physical methods deliver genetic material, such as naked DNA, through transient penetration of the cell membrane. The most studied of these methods include mechanical, electrical, hydrodynamic, ultrasonic, or magnetic force that have shown much promise. These techniques have minimal toxicity, and in some cases, have shown high levels of expression for periods lasting over 19 months in slow-dividing skeletal muscle. However, it is inherent in many cases that these physical techniques require invasive surgery and cause transient damage at the site of treatment. These techniques are briefly described below and will be covered in more detail in part II of this book. 


\subsection{Mechanical High-Pressure Delivery}

Mechanical high-pressure delivery, also referred to as hydrodynamic injection, was first demonstrated in 1999 by Dr. Feng Liu (Liu, Song, Zhang, \& Liu, 1999) and Dr. Guofeng Zhang (Zhang, Budker, \& Wolff, 1999). Gene expression in the liver, kidneys, lungs, and heart was demonstrated by rapid injection of a large volume of naked DNA solution into a mouse via the tail vein. The basic idea of hydrodynamic injection involves generating high pressure in a quick burst resulting in the formation of transient pores in the hepatocytes and subsequent diffusion of DNA into the cells. Hydrodynamic injection is considered to be the most efficient nonviral gene transfer method for in vivo gene delivery in mice. Although hydrodynamic injections show high levels of gene expression in small vertebrates, it is clear that this procedure will need significant modifications before advancing to the clinical setting with human patients. This procedure calls for large injection volumes which are deemed too great for human patients, and also causes transient damage to the target tissues. Improvements in this approach replace systemic injections with catheterization of the target tissues, allowing moderate injection volumes and computer-controlled injection rates. This approach has shown promising gene expression in large-animal studies (Suda, Suda, \& Liu, 2008). This improved technique could be the next step in introducing hydrodynamic injection-based gene delivery into clinical trials. Hydrodynamic injection is the most studied physical gene delivery method and is discussed further in part II, chapters 1 and 4.

\subsection{Electroporation-Mediated Delivery}

Electroporation-mediated delivery of genetic material was first applied to in vivo models in the early 1990s by Titomirov AV (Titomirov, Sukharev, \& Kistanova, 1991). This method is based on the use of applied electric fields to certain tissues in order to alter the cellular permeability. The formation of transient pores allows genetic material to diffuse through the cellular membrane and into the cell. The general procedure includes the injection of DNA into the target tissue, and subsequent electric force is applied allowing the genetic material to enter the cells. This technique seems to be a safer physical method of introducing genetic material to a tissue compared to hydrodynamic injections. Hashida's group used electroporation methods to achieve tissue specificity following a systemic injection in which high levels of targeted gene expression were found only where an electrical field was applied (Sakai, Nishikawa, Thanaketpaisarn, Yamashita, \& Hashida, 2005). Electroporation, discussed further in part II, chapters 1 and 3, has shown much promise with high levels of gene expression in specific targeted tissues, but like many physical methods, electroporation comes with some limitations. Placement of these electrodes requires surgery and in some cases, depending on the target organ, can be very difficult and invasive.

\subsection{Ultrasound-Mediated Delivery (Sonoporation)}

The use of ultrasound waves to disrupt the plasma membrane allowing material into the cell was first demonstrated in the early 1950s (Fry, Wulff, Tucker, \& Fry, 1950). The energy of the wave is absorbed by the tissue, creating abnormalities in the cell membrane which allows material access into the cytoplasm of the cell. The incorporation of microbubbles alongside ultrasound gene transfer has vastly improved this method of gene delivery. The microbubbles, which can be targeted to the desired tissue, act by absorbing the ultrasound 
waves, breaking apart, and releasing nearby shock waves which can cause the cell membrane to form transient pores. The size of the microbubbles and the agents used in forming these bubbles are critical in order to promote high gene expression. The efficiency of sonication-based gene delivery, discussed further in part II, chapters 1 and 2, is dependent on many factors. These factors include the frequency and intensity of the ultrasound wave, the presence of contrast agent, targeting ability of micro-bubbles, DNA concentration, and the duration of exposure (Bekeredjian, Grayburn, \& Shohet, 2005). Due to the safety and capability of targeting internal organs without surgical procedures, as well as the recent research of enhancing the permeability of the blood-brain barrier, ultrasound-mediated delivery has proved to be a less-invasive physical method. Although micro-bubbles and ultrasound bring improvements to the field of genetic material delivery, there are issues that need to be addressed. The first issue needing to be addressed is the protection of the genetic material against enzymes and shear forces in the body. Low gene expression levels compared to more invasive and extreme techniques such as electroporation and hydrodynamic injection is a drawback as well. Therefore, by better understanding the exact mechanism of action and optimizing the relationship between the micro-bubble construct and the ultrasound cavitation, this technique may start to see more promising preclinical and clinical results.

\subsection{Magnetic-Sensitive Nanoparticles (Magnetofection)}

In an attempt to address the transient damage caused by the invasive methods mentioned above (i.e., hydrodynamic injection and electroporation), magnetofection techniques have been introduced. This technique uses the physical method of a magnetic field to direct the deliver of genetic material to the desired target site. The concept involves attaching DNA to a magnetic nanoparticle usually consisting of a biodegradable substance such as iron oxide and coated with cationic polymer such as PEI (Mulens, Morales, \& Barber, 2013). These magnetic nanoparticles are then targeted to the tissue through a magnetic field generated by an external magnet. The magnetic nanoparticles are pulled into the target cells increasing the uptake of DNA. This technique is noninvasive and can precisely target the genetic material to the desired site while increasing gene expression. The drawback to magnetofection is the need to formulate magnetic nanoparticles complexed with naked DNA, as well as the need for strong external magnets.

\section{PERSPECTIVES}

The field of nonviral vectors has improved dramatically, gaining ground on the level of expression from viral gene delivery, while also addressing the safety issues that are analogous with these viral vectors. Nonviral vectors over the recent years have proved themselves successful in vivo results that generate therapeutically beneficial levels of expression. Although the transfection efficiency for these nonviral approaches is still well below that of the highly efficient viral vectors; it may not be necessary to achieve these high levels, as long as prolonged expression can be achieved. Further improvements to increase the prolonged expression (part II, chapters 5, 6, and 7) and reduce the toxicity of nonviral vectors (part I, chapter 12) will need to be addressed. In order to meet these needs, our knowledge and understanding of the mechanism of action of nonviral vectors as well as how 
viral genetic material can be preserved and expressed more efficiently must be improved. Understanding the viral pathway and incorporating the necessary material into a nonviral vector may be the necessary steps needed to successfully achieve a clinically revolutionary gene delivery system.

\section{Acknowledgments}

We dedicate this introduction chapter to the late Dr. Feng Liu, who coinvented the hydrodynamic method of gene transfer to the liver. Dr. Liu's inspiring and pioneering work has contributed greatly to the field of nonviral vector for gene therapy. The original work in authors' lab was supported by NIH grants CA149363, CA151652, and DK100664.

\section{References}

Bekeredjian R, Grayburn PA, Shohet RV. Use of ultrasound contrast agents for gene or drug delivery in cardiovascular medicine. Journal of the American College of Cardiology. 2005; 45:329-335. [PubMed: 15680708]

Farjo R, Skaggs J, Quiambao A, Cooper M, Naash M. Efficient non-viral ocular gene transfer with compacted DNA nanoparticles. PLoS One. 2006; 1:1-8.

Felgner PL, Gadek TR, Holm M, Roman R, Chan HW, Wenz M, et al. Lipofection: a highly efficient, lipid-mediated DNA-transfection procedure. Proceedings of the National Academy of Sciences of the United States of America. 1987; 84:7413-7417. [PubMed: 2823261]

Fry WJ, Wulff VJ, Tucker D, Fry FJ. Physical factors involved in ultrasonically induced changes in living systems: identification of non-temperature effects. The Journal of the Acoustical Society of America. 1950; 22:867-876.

Gaj T, Gersbach CA, Barbas CF 3rd. ZFN, TALEN, and CRISPR/Cas-based methods for genome engineering. Trends in Biotechnology. 2013; 31:397-405. [PubMed: 23664777]

Harvie P, Wong FM, Bally MB. Use of poly(ethylene glycol)-lipid conjugates to regulate the surface attributes and transfection activity of lipid-DNA particles. Journal of Pharmaceutical Sciences. 2000; 89:652-663. [PubMed: 10756331]

Hu Y, Haynes M, Wang Y, Liu F, Huang L. A highly efficient synthetic vector: nonhydrodynamic delivery of DNA to hepatocyte nuclei in vivo. ACS Nano. 2013; 7(6):5376-5384. [PubMed: 23647441]

Liu F, Song YK, Zhang G, Liu D. Hydrodynamics-based transfection in animals by systemic administration of plasmid DNA. Gene Therapy. 1999; 6:1258-1266. [PubMed: 10455434]

Li W, Szoka FC Jr. Lipid-based nanoparticles for nucleic acid delivery. Pharmaceutical Research. 2007; 24:438-449. [PubMed: 17252188]

Mintzer MA, Simanek EE. Nonviral vectors for gene delivery. Chemical Reviews. 2009; 109:259-302. [PubMed: 19053809]

Mulens V, Morales M, Barber D. Development of magnetic nanoparticles for cancer gene therapy: a comprehensive review. ISRN Nanomaterials, 2013. 2013; 14:646284.

Pack DW, Hoffman AS, Pun S, Stayton PS. Design and development of polymers for gene delivery. Nature Reviews Drug Discovery. 2005; 4:581-593. [PubMed: 16052241]

Sakai M, Nishikawa M, Thanaketpaisarn O, Yamashita F, Hashida M. Hepatocyte- targeted gene transfer by combination of vascularly delivered plasmid DNA and in vivo electroporation. Gene Therapy. 2005; 12:607-616. [PubMed: 15616599]

Suda T, Suda K, Liu D. Computer-assisted hydrodynamic gene delivery. Molecular Therapy. 2008; 16:1098-1104. [PubMed: 18398428]

Titomirov AV, Sukharev S, Kistanova E. In vivo electroporation and stable transformation of skin cells of newborn mice by plasmid DNA. Biochimica et Biophysica Acta. 1991; 1088:131-134.

[PubMed: 1703441] 
Wightman L, Kircheis R, Rössler V, Carotta S, Ruzicka R, Kursa M, et al. Different behavior of branched and linear polyethylenimine for gene delivery in vitro and in vivo. The Journal of Gene Medicine. 2001; 3:362-372. [PubMed: 11529666]

Yew NS, Zhao H, Wu IH, Song A, Tousignant JD, Przybylska M, et al. Reduced inflammatory response to plasmid DNA vectors by elimination and inhibition of immunostimulatory CpG motifs. Molecular Therapy. 2000; 1:255-262. [PubMed: 10933941]

Zhang G, Budker V, Wolff J. High levels of foreign gene expression in hepatocytes after tail vein injections of naked plasmid DNA. Human Gene Therapy. 1999; 10(10):1735-1737. [PubMed: 10428218] 

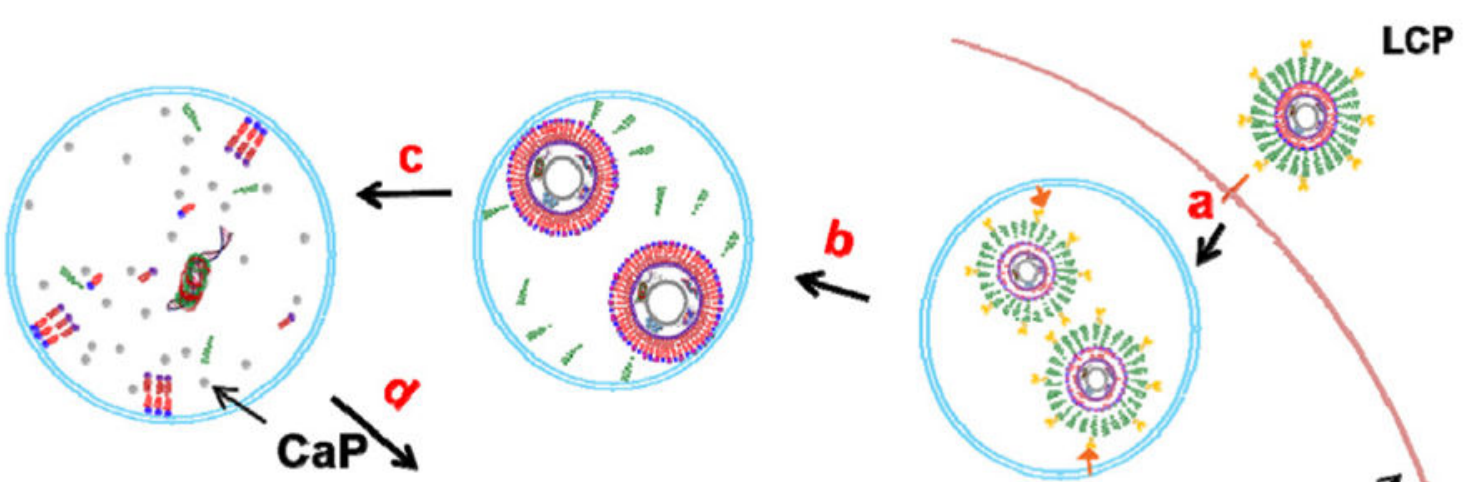

\section{nuclear membrane}

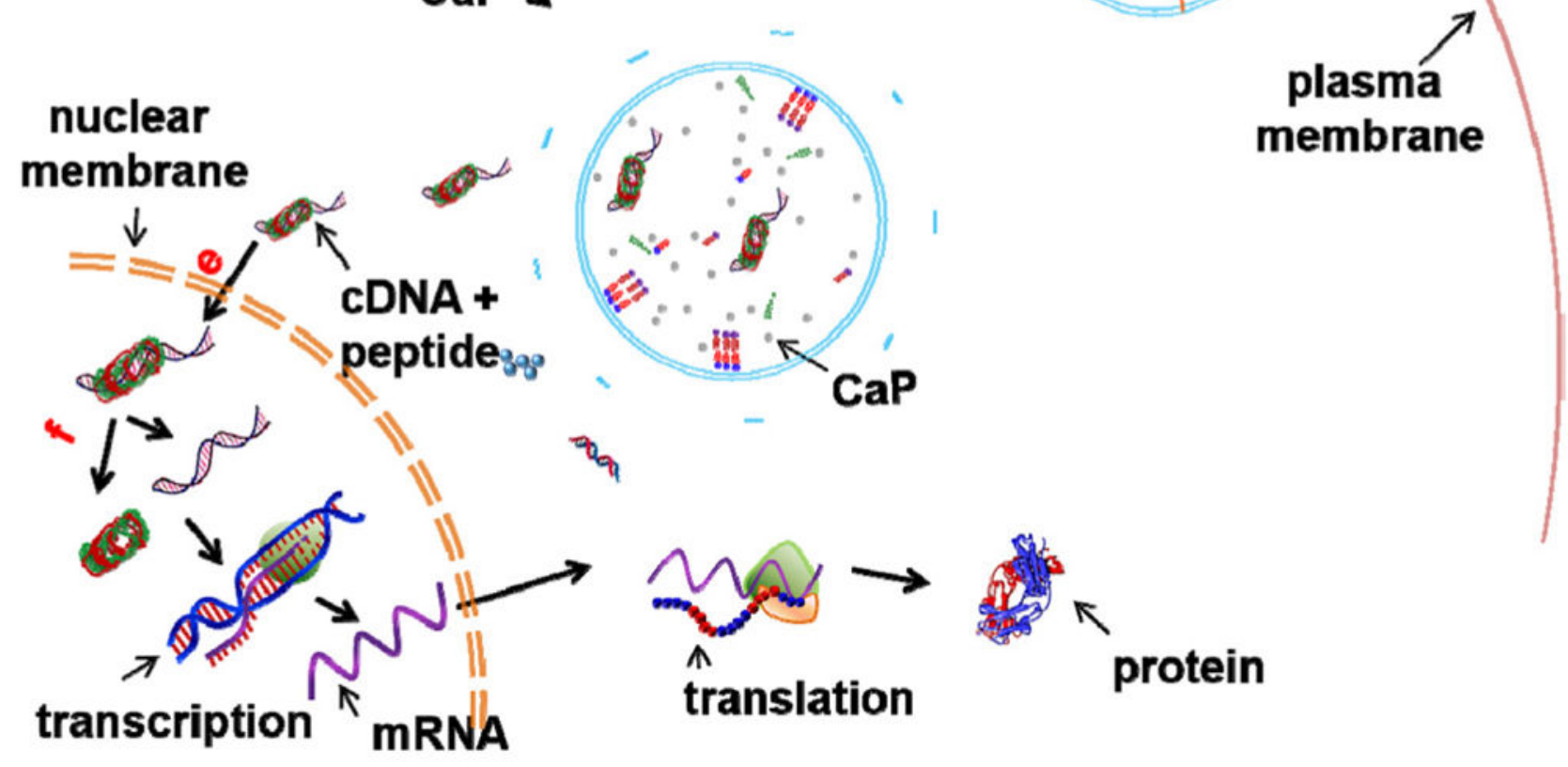

Figure 1.1. Proposed mechanism for intracellular delivery of DNA by lipid calcium phosphate (LCP)

Stepwise scheme for nonviral acid-sensitive vector (LCP), in which (a) the vector is internalized through receptor-mediated endocytosis, (b) PEG is shed from the vector, (c,d) vector and endosome further destabilized as endosome's $\mathrm{pH}$ decreases and releases the DNA-peptide complex into the cytoplasm. The DNA-peptide complex enters the nucleus through the nuclear pore, where it dissociates and releases free DNA, which is transcribed to mRNA, migrates to the cytoplasm to be translated, and results in desired protein synthesis (Hu et al., 2013). Original figure was prepared by Bethany DiPrete. (See the color plate.) 


\section{Table 1.1}

Comparison of improved hepatic luciferase gene expression in various nonviral gene delivery vectors (Hu et al., 2013)

\begin{tabular}{lll} 
Nonviral vector & Dose (mg DNA/kg) & Luc expression (RLU/mg protein) \\
\hline Poly(amine-co-ester) & 0.5 (i.t.) $^{\star}$ & $1.5 \star 10^{5}$ \\
Bifunctional dendrimer & 2.5 (i.v.) $^{\xi}$ & $7.5 \star 10^{5}$ \\
Ethyl-alkylated polyethylenimine & 2.5 (i.v.) & $1.0 \star 10^{6}$ \\
R8-GALA-MEND & 2.5 (i.v.) & $1.3 \star 10^{6}$ \\
LCP(mc-CR8C)Gal & 0.3 (i.v.) & $4.6 \star 10^{7}$ \\
Hydrodynamic injection & 0.3 (i.v.) & $4.8 \star 10^{9}$ \\
\hline${ }_{\text {Intratumoral tissue injection. }}$ & \\
$\mathcal{S}_{\text {Intravenous Injection. }}$ &
\end{tabular}

